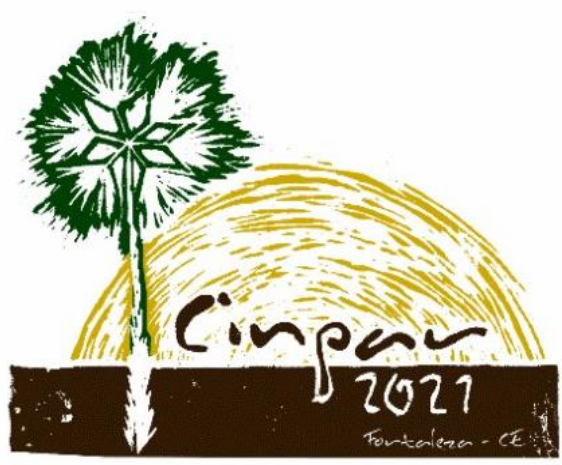

XVII Congresso Internacional sobre Patologia e

Reabilitação das Construções

XVII Congreso Internacional sobre Patología y Rehabilitación de las Construcciones

XVII International Conference on Pathology and Constructions Rehabilitation

FORTALEZA (Brasil), 3 a 5 de junho de 2021

https://doi.org/10.4322/CINPAR.2021.003

\title{
Dimensionamento ao cisalhamento de vigas reforçadas via a técnica NSM
}

\section{Shear dimensioning of reinforced beams the NSM technique}

\author{
Luis Henrique Pereira França ${ }^{1}$, André Luís Gamino ${ }^{2}$ \\ ${ }^{1}$ Universidade Federal do Ceará, Fortaleza, Brasil, luishenrique1924@hotmail.com \\ 2 Universidade de São Paulo, São Paulo, Brasil, andre.gamino@gmail.com
}

\begin{abstract}
Resumo: A falta de manutenções corretivas e preventivas em estruturas podem acarretar em manifestações patológicas que comprometem a vida útil da estrutura. Nesse sentido, o presente trabalho visou estudar a durabilidade e capacidade portante de vigas de concreto convencional submetida à flexão por meio da NBR 12142 (2010), para isto foi realizado reforço estrutural mediante a técnica Near Surface Mounted (NSM). As vigas podem romper por flexão, cisalhamento ou esmagamento do concreto, como o objetivo do presente trabalho é analisar a carga de ruptura frente à flexão, é necessário a armadura transversal, para que a viga rompa por flexão e não por cisalhamento. Tal reforço foi executado com chapas metálicas (A36) devido o seu baixo custo. Foram moldados 16 vigas em escala reduzida, o reforço foi incorporado no banzo tracionado com aplicação de resina epóxi. Após análise dos dados, conclui-se que a técnica e o material utilizado empregado aumentaram a capacidade portante da estrutura e tornou a ruptura mais ductil.
\end{abstract}

Palavras-chave: Reforço estrutural. NSM. Cisalhamento.

Abstract: The lack of corrective and preventive maintenance on structures can lead to pathological manifestations that compromise the useful life of the structure. In this sense, the present work aimed to study the durability and bearing capacity of conventional concrete beams submitted to bending by means of NBR 12142 (2010), for this structural reinforcement was performed using the Near Surface Mounted (NSM) technique. The beams can break by flexing, shearing or crushing the concrete, as the objective of the present work is to analyze the breaking load in relation to the bending, the transverse reinforcement is necessary, so that the beam breaks by bending and not by shearing. Such reinforcement was performed with metal plates (A36) due to its low cost. 16 beams were molded on a small scale, the reinforcement was incorporated in the tensioned flange with the application of epoxy resin. After analyzing the data, it is concluded that the technique and the material used employed increased the bearing capacity of the structure and made the rupture more ductile.

Keywords: Structural Reinforcement. NSM. Shearing. 


\section{Introdução}

Resistência e durabilidade são as propriedades principais do concreto armado, entretanto é do senso comum que as estruturas de concreto armado não necessitam de manutenções ao longo da sua vida útil. Segundo Al-Obaidi, Saeed e Rad (2020), as estruturas em concreto armado não são eternas e precisam de conservação para que suas características iniciais, as que foram dimensionadas, permaneçam durante sua vida útil de projeto. É do senso comum que as estruturas de concreto possuem vida útil ilimitada, o que colabora para falta de manutenções corretivas dispostas na NBR 15575, NBR 6118 e no Manual do Uso da Edificação entregue aos proprietários dos imóveis. As estruturas de concreto possuem vida útil projetada e limitada, para atingir a vida útil de projeto é necessário intervenções na estrutura ao longo do tempo.

Apesar da rápida execução e pouco prejuízo ao funcionamento das atividades, o que torna viável para aplicação em obras como viadutos, estacionamentos, edifícios e pontes. Ainda há algumas restrições para o uso das chapas como a espessura, excesso de umidade e temperatura que influência na perda de aderência da resina epóxi, a qual é responsável pela transferência de cargas. O material utilizado no reforço estrutural foi o aço, devido o seu baixo custo e a alta disponibilidade na região, entretanto não há muitos estudos com a aplicação de chapas metálicas, há muitos trabalhos com FRP, logo estes serviram como base para melhor entendimento da técnica.

Como é descrito por Dias, Silva e Barros (2021), o reforço caracteriza-se como uma atividade específica para os casos em que se deseja melhorar o desempenho de uma estrutura, dotando-a de maior resistência e/ou rigidez. Os problemas patológicos na maioria dos casos apresentam características externas como: manchas, corrosão das armaduras, fissuras ativas e passivas, degradação química e ninhos. As manifestações patológicas podem ocorrer devido ao mau uso, falta de planejamento, erro projeto e má qualidade dos materiais empregados (HELENE, 1988). Nesse sentido, o presente estudo visou estudar a técnica NSM com embutidura de chapas metálicas no banzo tracionado para analisar a carga de ruptura e dimensionamento ao cisalhamento mudando a profundidade do entalhe e o espaçamento entre os mesmos. Nesse sentido, o objetivo desse estudo foi o de analisar o processo de ruptura e o aumento da capacidade portante com a inserção de reforço via a técnica NSM com chapas metálicas.

\section{Revisão de literatura}

Diante da necessidade de estudar e melhorar a capacidade resistente das edificações existentes, já no século passado iniciaram os estudos de reabilitação estrutural mediante a técnica NSM, sendo amplamente abordado em países europeus e norte-americanos. Normalmente é utilizado tanto em vigas, lajes e pilares para reforço à flexão e ao cisalhamento (ASKANDAR; MAHMOOD, 2019). A técnica apresenta resultados positivos no aumento da resistência de capacidade de carga e, bem como, em ductilidade da estrutura. Como aponta Dias e Barros (2005), a configuração da técnica NSM é condicionada pela espessura do cobrimento, como pode ser visto na Figura 1.

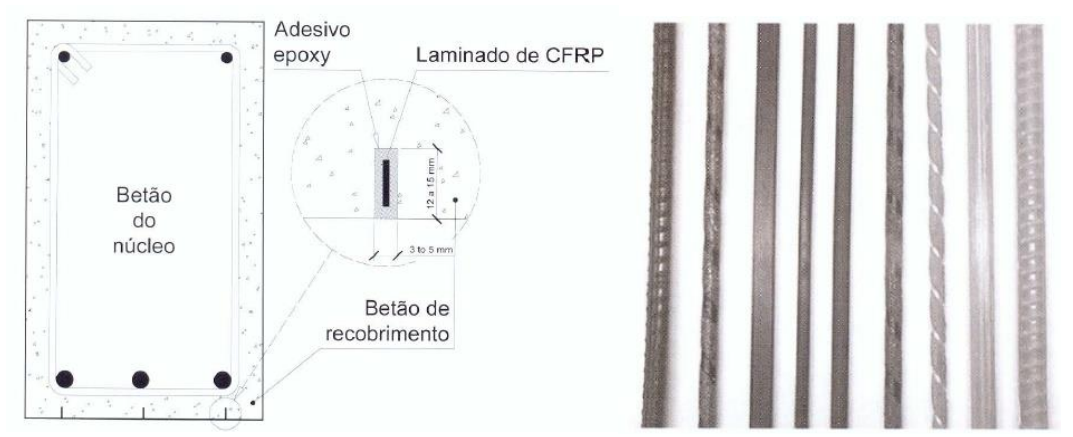

Figura 1 - Disposição do corte realizado no concreto (CRUZ, 2008).

Há divergências sobre a espessura e profundidade do corte a ser realizado na estrutura, Cruz (2008) retrata que as ranhuras devem ser quadradas ou retangulares, mas com espessuras entre $1 \mathrm{~mm}$ a $4 \mathrm{~mm}$. Para Fortes 
et al. (2003), os entalhes devem possuir de $3 \mathrm{~mm}$ a $4 \mathrm{~mm}$ de espessura e $12 \mathrm{~mm}$ de profundidade, Azevedo (2008), propõe espessuras entre $3 \mathrm{~mm}$ a $5 \mathrm{~mm}$ e profundidade entre $12 \mathrm{~mm}$ a $15 \mathrm{~mm}$. Segundo Almeida (2013), as dimensões dessa técnica quanto a laminados podem variar de 1,0 a 1,5 mm de espessura e de 10 $\mathrm{mm}$ a $15 \mathrm{~mm}$ de largura. Porém, alguns autores indicam valores de largura até $30 \mathrm{~mm}$, em relação os vergalhões essas dimensões são variadas. Alguns aspectos de aplicação desse reforço: usado em superfícies planas, não obrigatoriedade da retirada da nata de cimento, possibilidade de colagem com argamassa, dependente da espessura do cobrimento, antes da colagem é imprescindível a limpeza das ranhuras, indicado para reforços à flexão (momentos positivos e negativos) e cisalhamento, não é necessária proteção quanto a atos de vandalismo, maior resistência ao fogo e condições ambientais (SHAKIR; KAMONNA, 2018).

\section{Materiais e métodos}

Baseando-se nos critérios da NBR 6118 (2014) e NBR 12142 (2010), foi possível analisar os resultados obtidos nos ensaios de tração na flexão, dando ênfase no aumento da resistência da carga de ruptura e redução da taxa de armadura transversal. Foram realizados ensaios experimentais com vigas fletidas em escala reduzida $(0,15 \times 0,15 \times 0,55 \mathrm{~m})$ com uso de chapas metálicas, após os ensaios houve mais clareza dos benefícios e malefícios que a técnica de reabilitação causa na estrutura. Foram realizadas quatro séries de moldagens, sendo que para cada moldagem dois corpos de prova prismáticos ficaram como referência. Foi realizada a criação da ranhura e a colagem do reforço com resina à base epóxi (Figura 2). 0 ensaio de tração na flexão foi realizado em consonância com a NBR 12142 (2010), como pode ser visto na Figura 3.
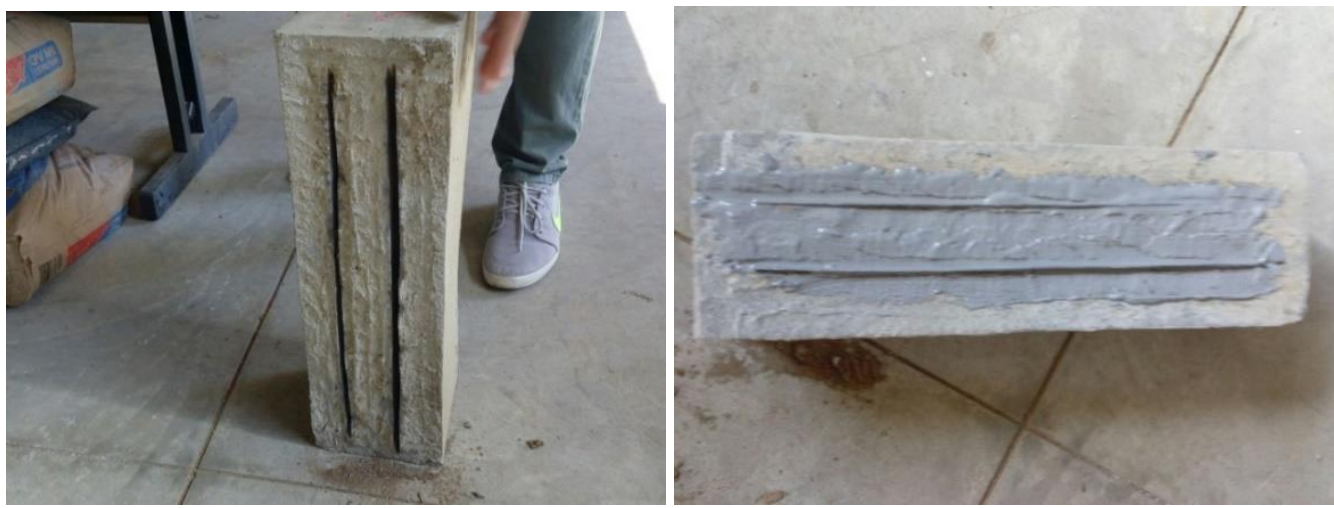

Figura 2 - Criação dos cortes e colocação dos laminados nas vigas.

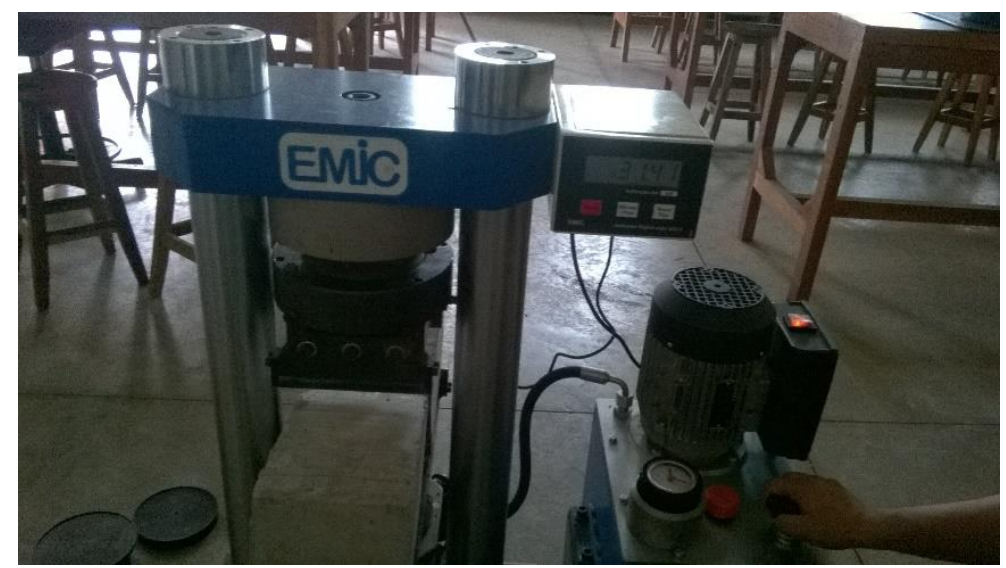

Figura 3 - Modelo de ensaio realizado.

Dimensionamento ao cisalhamento das vigas em escala reduzida: as vigas foram dimensionadas de acordo com a NBR 6118 (2014), levando em consideração sua resistência à compressão, tração e taxa de armaduras, obedecendo os seguintes passos: 
10 passo: Cálculo do $f_{c t m}$ do ensaio de tração na flexão

- $\quad$ $f_{c t m}$ obtido com base na expressão abaixo:

$$
f_{c t m}=0,7 \cdot f_{c t, f \text { média }}
$$

Para evitar uma ruptura prematura e viabilizar que as tensões de compressão continuem atuando é recomendado a colocação de armadura transversal.

20 passo: Dimensionamento da armadura transversal

- Verificação da biela comprimida:

$$
\begin{array}{r}
V_{R d 2}=0,27 \cdot \alpha_{v} \cdot f_{c d} \cdot b_{w} \cdot d \\
V_{c 0}=0,6 \cdot f_{c t d} \cdot b_{w} \cdot d
\end{array}
$$

- Verificação da Armadura Mínima

$$
\left(\frac{A_{s w}}{s}\right)_{\min }=\rho_{s w, \min } \cdot b_{w}
$$

- Método econômico:

$$
\begin{gathered}
V_{s w}=\left(\frac{A_{s w}}{s}\right)_{\min } \cdot 0,9 \cdot d \cdot f_{y w d} \\
V_{R d 3}=V_{c}+V_{s w}
\end{gathered}
$$

Para melhor dimensionamento e diminuição do custo, procurou-se colocar o $V_{R d 3}$ dentro dos limites dos diagramas de esforço cortante obtidos via FTOOL. Logo, caracteriza-se armadura mínima para o trecho do diagrama de cortante onde $V_{\mathrm{Rd} 3}$ seja maior que o $\mathrm{V}_{\mathrm{sd}}$.

3ำ passo: Verificação da bitola e espaçamento:

$$
\text { Bitolas } \rightarrow 5 \mathrm{~mm} \leq \emptyset \leq 15 \mathrm{~mm}
$$

- Espaçamento dos estribos (S)

$$
\mathrm{S}_{\min }=7 \mathrm{~cm} ; \mathrm{S}_{\operatorname{máx}}=0,6 \cdot d \leq 30 \mathrm{~cm}
$$

- Verificação dos espaçamentos:

$$
S(m)=\frac{2 A_{s 1}}{\left(\frac{A_{S w}}{s}\right)}
$$

Fazendo a razão entre o comprimento da viga pelo espaçamento entre estribos, foi possível chegar à quantidade de estribos necessários para armar esta viga para que não se rompa por efeito cisalhante, uma Dimensionamento ao cisalhamento de vigas reforçadas via a técnica NSM 
vez que o presente estudo visou estudar o comportamento à flexão, o dimensionamento ao cisalhamento foi realizado para melhor entendimento da ruptura do elemento estrutural na flexão.

\section{Resultados e discussões}

Primeiramente foi analisada a forma que se deu a ruptura da viga, uma vez que o objeto em análise do presente trabalho é o reforço à flexão, logo a viga não poderia se romper por cisalhamento ou esmagamento do concreto. Notou-se processos de rupturas diferentes, por exemplo, na Figura 4 e 5 nitidamente tem processos de rupturas distintas, a primeira sofre ruptura por cisalhamento e a outra por flexão. A ruptura por flexão ocorre no centro da viga, logo foi realizado o dimensionado ao cisalhamento para que a viga se rompa por flexão e não por cisalhamento.

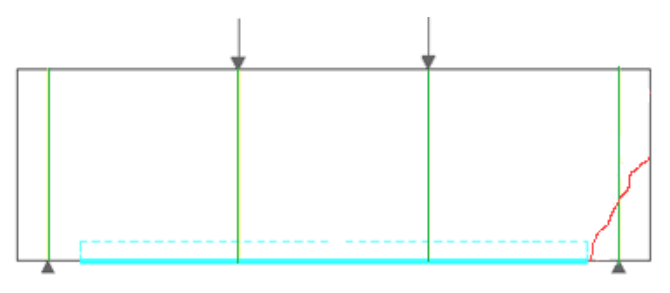

Demarcação dos terços

Chapa metálica

Modelo aproximado da fissura encontrada nos ensaios

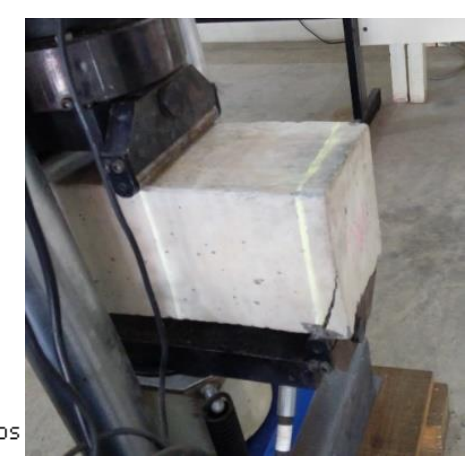

Figura 4 - Corpo prismático reforçado com NSM (espaçamento de $3 \mathrm{~cm}$ entre as lâminas.

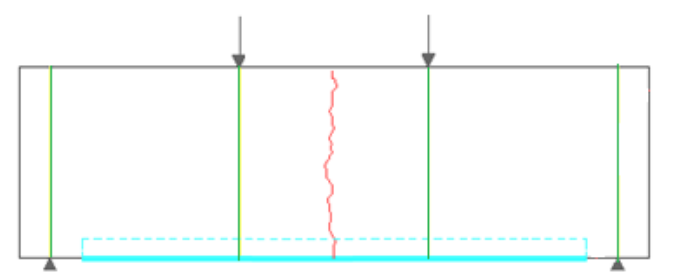

Demarcação dos terços

Chapa metálica

Modelo aproximado da fissura encontrada nos ensaios

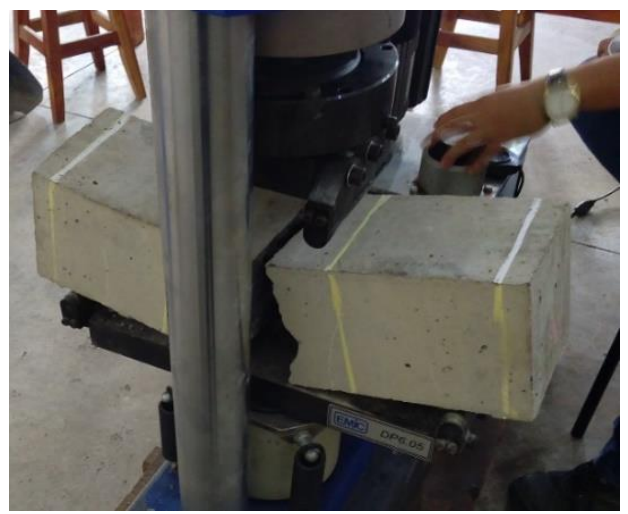

Figura 5 - Corpo prismático reforçado com NSM (espaçamento de $5 \mathrm{~cm}$ entre as lâminas

Nos Quadros 1,2,3 e 4 abaixo encontram-se o dimensionamento ao cisalhamento das vigas não reforçadas e reforçadas. As primeiras e segundas colunas de todas as tabelas referem-se aos dados obtidos nos ensaios de tração na flexão conforme NBR 12142 (2010), as demais colunas referem-se ao dimensionamento analítico das vigas, conforme a NBR 6118 (2014). As duas primeiras linhas de cada tabela referem-se às vigas de referências (sem reforço), e as demais de vigas reforçadas. 
Quadro 1 - Dimensionamento ao cisalhamento das vigas reforçadas via técnica NSM, com profundidade de $2 \mathrm{~cm}$ e espaçamento de $5 \mathrm{~cm}$.

\begin{tabular}{|c|c|c|c|c|c|c|c|c|}
\hline Elementos & $\begin{array}{c}\text { Força de } \\
\text { Ruptura } \\
\text { (kN) }\end{array}$ & $\mathrm{V}_{\mathrm{sd}}(\mathrm{kN})$ & $\begin{array}{l}V_{\mathrm{Rd} 2} \\
(\mathrm{kN})\end{array}$ & $\mathrm{V}_{\mathrm{c}}(\mathrm{kN})$ & $V_{s w}(k N)$ & $\begin{array}{l}V_{\text {Rd3 }} \\
(k N)\end{array}$ & $\left(\frac{A_{s w}}{s}\right)_{m i ́ n}$ & $\left(\frac{A_{s w}}{S}\right)$ \\
\hline V1N52 & 32,26 & 22,27 & 87,83 & 25,25 & 13,20 & 38,45 & $\begin{array}{c}6 \varphi 5,0 \mathrm{~mm} \mathrm{c} / \\
8 \mathrm{~cm}\end{array}$ & - \\
\hline V2N52 & 21,11 & 14,96 & 87,83 & 25,25 & 13,20 & 38,45 & $\begin{array}{c}6 \varphi 5,0 \mathrm{~mm} \mathrm{c} / \\
8 \mathrm{~cm}\end{array}$ & - \\
\hline V3R52 & 62,40 & 43,87 & 87,83 & 25,25 & 18,62 & 43,87 & $\begin{array}{c}4 \varphi 5,0 \mathrm{~mm} \mathrm{c} / \\
8 \mathrm{~cm}\end{array}$ & $\begin{array}{c}2 \varphi 5,0 \\
\mathrm{~mm} \mathrm{c} / 8 \\
\mathrm{~cm}\end{array}$ \\
\hline V4R52 & 62,83 & 44,17 & 87,83 & 25,25 & 18,92 & 44,17 & $\begin{array}{c}4 \varphi 5,0 \mathrm{~mm} \mathrm{c} / \\
8 \mathrm{~cm}\end{array}$ & $\begin{array}{c}2 \varphi 5,0 \\
\mathrm{~mm} \mathrm{c} / 8 \\
\mathrm{~cm}\end{array}$ \\
\hline
\end{tabular}

Quadro 2 - Dimensionamento ao cisalhamento das vigas reforçadas via técnica NSM, com profundidade de $2 \mathrm{~cm}$ e espaçamento de $3 \mathrm{~cm}$.

\begin{tabular}{|c|c|c|c|c|c|c|c|c|}
\hline Elementos & $\begin{array}{c}\text { Força de } \\
\text { Ruptura } \\
\text { (kN) }\end{array}$ & $\begin{array}{l}V_{\text {sd }} \\
(\mathbf{k N})\end{array}$ & $\begin{array}{l}V_{\text {Rd2 }} \\
(k N)\end{array}$ & $\mathrm{V}_{\mathrm{c}}(\mathrm{kN})$ & $\begin{array}{l}V_{\text {sw }} \\
(k N)\end{array}$ & $\begin{array}{l}V_{\text {Rd3 }} \\
(k N)\end{array}$ & $\left(\frac{A_{s w}}{s}\right)_{m i ́ n}$ & $\left(\frac{A_{s w}}{s}\right)$ \\
\hline V5N32 & 37,22 & 26,25 & 87,83 & 25,64 & 13,38 & 39,02 & $\begin{array}{c}6 \varphi 5,0 \mathrm{~mm} \mathrm{c} / \\
8 \mathrm{~cm}\end{array}$ & - \\
\hline V6N32 & 43,36 & 30,55 & 87,83 & 25,64 & 13,38 & 39,02 & $\begin{array}{c}6 \varphi 5,0 \mathrm{~mm} \mathrm{c} / \\
8 \mathrm{~cm}\end{array}$ & - \\
\hline V7R32 & 49,52 & 34,82 & 87,83 & 25,64 & 13,38 & 39,02 & $\begin{array}{c}6 \varphi 5,0 \mathrm{~mm} \mathrm{c} / \\
8 \mathrm{~cm}\end{array}$ & - \\
\hline V8R32 & 51,00 & 35,90 & 87,83 & 25,64 & 13,38 & 39,02 & $\begin{array}{c}6 \varphi 5,0 \mathrm{~mm} \mathrm{c} / \\
8 \mathrm{~cm}\end{array}$ & - \\
\hline
\end{tabular}

Quadro 3 - Dimensionamento ao cisalhamento das vigas reforçadas via técnica NSM, com profundidade de $3 \mathrm{~cm}$ e espaçamento de $5 \mathrm{~cm}$.

\begin{tabular}{|c|c|c|c|c|c|c|c|c|}
\hline Elementos & $\begin{array}{c}\text { Força de } \\
\text { Ruptura } \\
\text { (kN) }\end{array}$ & $V_{s d}(k N)$ & $\begin{array}{l}V_{\text {Rd2 }} \\
\text { (kN) }\end{array}$ & $\mathrm{V}_{\mathrm{c}}(\mathrm{kN})$ & $\mathrm{V}_{\mathrm{sw}}(\mathrm{kN})$ & $\begin{array}{l}V_{\mathrm{Rd} 3} \\
(\mathrm{kN})\end{array}$ & $\left(\frac{A_{s w}}{S}\right)_{\min }$ & $\left(\frac{A_{s w}}{s}\right)$ \\
\hline V9N53 & 32,23 & 22,75 & 87,83 & 27,33 & 14,27 & 41,60 & $\begin{array}{c}6 \varphi 5,0 \mathrm{~mm} \mathrm{c} / \\
8 \mathrm{~cm}\end{array}$ & - \\
\hline V10N53 & 30,60 & 21,61 & 87,83 & 27,33 & 14,27 & 41,60 & $\begin{array}{c}6 \varphi 5,0 \mathrm{~mm} \mathrm{c} / \\
8 \mathrm{~cm}\end{array}$ & - \\
\hline V11R53 & 59,54 & 41,87 & 87,83 & 27,33 & 14,54 & 41,87 & $\begin{array}{c}6 \varphi 5,0 \mathrm{~mm} \mathrm{c} / \\
8 \mathrm{~cm}\end{array}$ & - \\
\hline V12R53 & 70,91 & 49,83 & 87,83 & 27,33 & 22,50 & 49,83 & $\begin{array}{c}4 \varphi 5,0 \mathrm{~mm} \mathrm{c} / \\
8 \mathrm{~cm}\end{array}$ & $\begin{array}{c}2 \varphi 5,0 \\
\mathrm{~mm} \mathrm{c} / 8 \\
\mathrm{~cm}\end{array}$ \\
\hline
\end{tabular}


Quadro 4 - Dimensionamento ao cisalhamento das vigas reforçadas via técnica NSM, com profundidade de $3 \mathrm{~cm}$ e espaçamento de $3 \mathrm{~cm}$.

\begin{tabular}{|c|c|c|c|c|c|c|c|c|}
\hline Elementos & $\begin{array}{c}\text { Força } \\
\text { de } \\
\text { Ruptura } \\
\text { (kN) }\end{array}$ & $\begin{array}{c}V_{\text {sd }} \\
(k N)\end{array}$ & $\begin{array}{l}V_{\text {Rd2 }} \\
\text { (kN) }\end{array}$ & $\begin{array}{c}V_{c} \\
(k N)\end{array}$ & $\begin{array}{l}V_{\text {sw }} \\
\text { (kN) }\end{array}$ & $\begin{array}{l}V_{\text {Rd3 }} \\
\text { (kN) }\end{array}$ & $\left(\frac{A_{s w}}{S}\right)_{\min }$ & $\left(\frac{A_{s w}}{s}\right)$ \\
\hline V13N33 & 30,72 & 21,70 & 87,83 & 23,87 & 12,47 & 36,34 & $\begin{array}{c}6 \varphi 5,0 \\
\mathrm{~mm} \mathrm{c} / 8 \\
\mathrm{~cm}\end{array}$ & - \\
\hline V14N33 & 29,45 & 20,81 & 87,83 & 23,87 & 12,47 & 36,34 & $\begin{array}{c}6 \varphi 5,0 \\
\mathrm{~mm} \mathrm{c} / 8 \\
\mathrm{~cm}\end{array}$ & - \\
\hline V15R33 & 52,80 & 37,16 & 87,83 & 23,87 & 13,29 & 39,16 & $\begin{array}{c}6 \varphi 5,0 \\
\mathrm{~mm} \mathrm{c} / 8 \\
\mathrm{~cm}\end{array}$ & - \\
\hline V16R33 & 55,41 & 38,98 & 87,83 & 23,87 & 15,11 & 38,98 & $\begin{array}{c}6 \varphi 5,0 \\
\mathrm{~mm} \mathrm{c} / 8 \\
\mathrm{~cm}\end{array}$ & - \\
\hline
\end{tabular}

Analisando todos os dimensionamentos alguns apontamentos podem ser feitos, tais como: quanto maior a carga de ruptura, menor será o espaçamento entre os estribos; o acréscimo da área de reforço não significa ganho de resistência mecânica; o aumento da capacidade resistente à flexão pode levar a uma ruptura por cisalhamento; não foi possível aproveitar toda a capacidade resistente da chapa, pois o concreto rompia antes de houver descolamento ou fratura da chapa; o uso de distâncias desiguais na inserção da chapa com a técnica NSM configurou na redução da carga de ruptura.

O dimensionamento ao cisalhamento foi realizado, pois o reforço estrutural tem como função atuar como uma armadura longitudinal e, em alguns casos, até como armadura de bordo. Logo, os estribos foram calculados para obter uma relação da taxa de armadura, visando comparar a área de reforço com a carga de ruptura, para dar direcionamento em futuras pesquisas. Percebeu-se nesse estudo que as armaduras transversais em quase todos os casos foram armadura mínima, espera-se que quando extrapolado para vigas em tamanhos reais, pode-se utilizar uma armadura transversal única ao longo de todo o vão.

A técnica NSM apresentou ótimo desempenho, pois apresentou várias características que justifica sua utilização, tais como: alto ganho de resistência mecânica, menor área de reforço e ruptura avisada. Entretanto, a criação dos cortes de forma uniforme em ambiente de difícil acesso pode ser sua principal desvantagem. Com o uso da técnica houve melhor aproveitamento do material de reforço, boa aderência, ruptura dúctil e ganho de resistência, tudo isso com uma área de reforço, relativamente, pequena.

\section{Conclusões}

O reforço estrutural apresentou resultados atrativos quanto a ganho de resistência mecânica, com valores máximos de ganho de resistência de $134 \%$, quando comparado a viga reforçada com a viga referência, usando pouco material de reforço, por intermédio do modelo com espaçamento equidistante de $5 \mathrm{~cm}$ entre lâminas e profundidade de $2 \mathrm{~cm}$. Além do aumento da resistência mecânica, a técnica NSM mostrou alguns pontos relevantes que justificam seu uso e se destaca como melhor técnica estudada, como: aproveitamento do material de reforço, aumento significativo da capacidade portante da viga reforçada, ruptura dúctil e avisada, maior contato reforço-substrato e redução da área de reforço. 
Outro fator vantajoso que justifica o uso da técnica NSM é a maior possibilidade de visualização de futuras fissuras no banzo tracionado. Em meio a todos os fatores e antes de qualquer tomada de decisão, é de suma importância o conhecimento prévio da estrutura, suas necessidades e deficiências, se há a possibilidade da paralização do uso, disponibilidade do material usado como reforço, agressividade do meio, dentre outros pontos que determinam a viabilidad, só assim há garantias da real eficácia e o melhor custo benefício da técnica a ser usada.

\section{Referências}

ALMEIDA, J. P. S. Comportamento estrutural a longo prazo e de durabilidade de elementos de concreto reforçados de acordo com a técnica NSM. Dissertação (mestrado em engenharia civil) - Universidade do Minho, Braga, 2013.

AL-OBAIDI, Salam; SAEED, Yasir M.; RAD, Franz N. Flexural strengthening of reinforced concrete beams with NSM CFRP bars using mechanical interlocking. Journal of Building Engineering, v. 31, p. 101422, 2020.

ASKANDAR, Nasih; MAHMOOD, Abdulkareem. Comparative investigation on torsional behaviour of RC beam strengthened with CFRP fabric wrapping and near-surface mounted (NSM) steel bar. Advances in Civil Engineering, v. 2019, 2019.

ASSOCIAÇÃO BRASILEIRA DE NORMAS TÉCNICAS. NBR 6118: Projeto de estruturas de concreto Procedimento. 2014, $221 \mathrm{p}$.

NBR 12142: Determinação da resistência à tração na flexão de corpos de prova prismáticos. Rio de Janeiro, 2010. 5 p.

AZEVEDO, D. M. M. Reforço de estruturas de concreto com colagem de sistemas compósitos CFRP - Tese (mestrado em engenharia civil) - Faculdade de engenharia civil da universidade do Porto, Porto, 2008.

CRUZ, J. S. Técnica NSM - Uma nova abordagem de estruturas com materiais compósitos. Revista Construção Magazine, v.28, p. 27-30, set. 2008.

DIAS, S. J .E. ; BARROS, J. A. O. Reforço ao corte de vigas T de concreto armado usando a técnica NSM com laminado de CFRP. In: CONGRESSO BRASILEIRO DO CONCRETO, 47, Anais... Pernambuco, Olinda, 2005.

DIAS, S. J. E.; SILVA, J. R. M.; BARROS, J. A. O. Flexural and shear strengthening of reinforced concrete beams with a hybrid CFRP solution. Composite Structures, v. 256, p. 113004, 2021.

FORTES, A. S.; BARROS, J. A. O. de; PADARATZ, I. J. Estudo comparativo de três técnicas de reforço à flexão com CFRP. In: CONGRESSO BRASILEIRO DE CONCRETO, 45. 2003. Vitória, ES. Anais... Vitória: IBRACON, 2003.

HELENE, P. R. L. Manual prático para reparo e reforço de estruturas de concreto. São Paulo: PINI, 1988. $119 p$.

SHAKIR, Qasim M.; KAMONNA, Hayder HH. The Behavior of High Strength Self-Compacting Reinforced Concrete Corbels Strengthened with NSM Steel Bars. Int. J. Adv. Sci. Eng. Inf. Technol, v. 8, n. 4, p. 1022$1028,2018$. 